\title{
Shape optimization of microstructural designs subject to local stress constraints within an XFEM-level set framework
}

\author{
Lise Noël · Pierre Duysinx
}

Received: date / Accepted: date

\begin{abstract}
The present paper investigates the tailoring of bimaterial microstructures minimizing their local stress field exploiting shape optimization. The problem formulation relies on the extended finite element method (XFEM) combined with a level set representation of the geometry, to deal with complex microstructures and handle large shape modifications while working on fixed meshes. The homogenization theory, allowing extracting the behavior of periodic materials built from the repetition of a representative volume element (RVE), is applied to impose macroscopic strain fields and periodic boundary conditions to the RVE. Classical numerical homogenization techniques are adapted to the selected XFEM-level set framework. Following previous works on analytical sensitivity analysis [31], the scope of the developed approach is extended to tackle the problem of stress objective or constraint functions. Finally, the method is illustrated by revisiting 2D classical shape optimization examples: finding the optimal shapes of single or multiple inclusions in a microstructure while minimizing its local stress field.
\end{abstract}

Keywords Microstructural design - Shape optimization · XFEM · Level set · Stress minimization · Homogenization

The first author, Lise Noël, is supported by a grant from the Belgian National Fund for Scientific Research (F.R.S.-FNRS) which is gratefully acknowledged.

Lise Noël

University of Liège, Aerospace and Mechanics,

Allée de la Découverte, 13A, B52, 4000 Liège

E-mail: Lise.Noel@ulg.ac.be

Pierre Duysinx

University of Liège, Aerospace and Mechanics,

Allée de la Découverte, 13A, B52, 4000 Liège

E-mail: p.duysinx@ulg.ac.be

\section{Introduction}

Nowadays, there is a huge demand for highly efficient materials to fulfill some particular requirements, even contradictory ones. In fact, with the development of the automotive, transport, space... industries, the interest in new materials exhibiting extreme properties is greater than ever. Therefore, many research works are devoted to the tailoring of material suited for specific applications. The macroscopic behavior of materials is related to their microstructures. It follows that designing material at their microstructural level allows obtaining prescribed behaviors at the macroscopic level.

In the 1970s, the homogenization theory was developed to bind the local and global behaviors of materials. In general, periodic materials are studied, i.e. materials built from the periodic repetition of a representative volume element (RVE) in the two (resp. three) directions of space. Effective macroscopic properties can be extracted starting from the microstructural specificities assuming that the RVE scale is very small compared to the macroscopic one, i.e. there is a clear separation between these two scales. The theory of homogenization is detailed by several authors as Bensoussan et al. [7], Sanchez-Palencia [35], Suquet [40] or Torquato [43]. Later on, to tackle bigger and more complex microstructural problems, numerical homogenization methods, resorting to the finite element method (FEM), were developed. The latter methods are described by Guedes and Kikuchi [15] or more recently by Andreassen and Andreasen [3].

Homogenization plays a key role in many engineering problems. In particular, interaction between homogenization theory and topology optimization is rather important. This interaction is bidirectional. In their seminal work, Bendsøe and Kikuchi [4] developed an 
approach to provide optimal structural topologies by searching for an appropriate material distribution over a given design domain. To circumvent the combinatorial problem of presence/absence of material at a point, they relaxed the problem by introducing porous materials in the formulation. The effective properties of the porous periodic microstructure were evaluated using the homogenization theory. The approach was further extended by several authors such as Suzuki and Kikuchi [41] for bending problems or Diaz and Kikuchi [10] for eigenfrequency maximization. Several microstructural geometries have been considered for instance square holes in Bendsøe and Kikuchi [4], rank-N materials in Bendsøe [6] or Allaire and Kohn [2]. Even if optimal stiffness properties are obtained as for rank-N materials, there is no consideration of the local stress field in the microstructures and so no consideration of the strength of microstructured materials. Reentrant corners in square holed microstructures and connections between layers in rank-2 materials, as shown in Fig.(1), clearly exhibit infinite stresses.

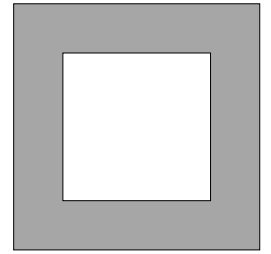

(a) Square holed microstructure.

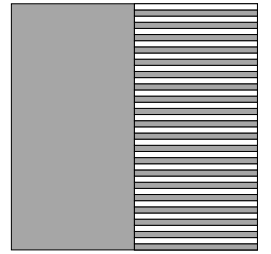

(b) Rank-2 microstructure.
Fig. 1 Microstructures optimized for stiffness and exhibiting infinite stresses at reentrant corners $(1(\mathrm{a}))$ or at connections between material layers (1(b)).

On the other way around, optimization has been extensively used to design material microstructural layouts exhibiting some prescribed macroscopic properties. The material design problem, known as material tailoring or inverse homogenization, can be solved efficiently by resorting to topology optimization tools as shown by Sigmund [37], [38]. This initial work was later followed by numerous others considering various objectives: extreme thermal expansion as in Sigmund and Torquato [39], maximized stiffness as in Guest and Prevost [16] or Weihong et al. [51], extremal bulk modulus as in Gibiansky and Sigmund [13] or Andreasen et al. [36]. A review of homogenization and topology optimization is given by Hassani and Hinton [18], [19], [20]. Shape optimization was also exploited to design materials assuming given parametric geometrical shapes as, for example, in Zhou et al. [52] or Procházka and Válek
[33] where the optimal shapes of short fibers maximizing the bearing capacity of composites were studied.

Extending topology optimization scope to account for local stress constraints, Duysinx and Bendsøe [12] assumed that stress constraints could be considered at the microstructural level by detecting the first point exceeding the limit resistance of the material. After investigating the stress level in rank-2 microstructures, a macroscopic failure model for porous microstructures was assumed. The corollary of the latter approach is to investigate layouts and geometries able to minimize the local stress state in a microstructure for a given macroscopic stress or strain state. However, despite the growing number of works on microstructural designs, local and global stress are scarcely used as objective or constraint functions.

Early works by Vigdergauz [47], [49], [48] provide some analytical and numerical solutions for the design of microstructures of maximal stiffness. Later on, these results were extended by Grabovsky and Kohn [14] showing that the Vigdergauz microstructure was actually minimizing the stress concentrations. More recently, Lipton [22] and Lipton and Stuebner [23], [24] provided some analytical and numerical results for functionally graded composites and for composite structures undergoing stress constraints. They proposed an inverse homogenization approach based on the minimization of some modulation functions connecting the macroscopic stress to the local stress fluctuations at microscale. More recently, Najafi et al. [30] also proposed an inverse homogenization problem to design particulate composites considering a potential damage in the inclusions using a NURBS-based shape optimization scheme. They seek for an appropriate layout of the composite which minimizes the area between the effective stress-strain curve and a prescribed non linear curve corresponding to a particular behavior.

The present work aims at contributing to restart an investigation of the stress state at microstructural level. In this initial work, we adopt a numerical shape optimization approach to design bimaterial microstructures minimizing their local stress fields under given macroscopic strain state. The level set method is used to represent the microstructure geometry allowing us to deal with complex shapes as well as with large shape modifications. To avoid difficulties related to classical shape optimization as frequent remeshing operations, the analysis is carried out on a fixed mesh resorting to the extended finite element method (XFEM). The method can handle discontinuities across material interfaces within the elements by adding some enriched shape functions to the classical FEM approximations. Exploiting this XFEM-level set framework provides an 
accurate knowledge of the local stress field around the interfaces and thus a fine control on the local stress. The sensitivity analysis is performed through an analytical approach previously developed by Noël et al. [31] and extended to the evaluation of stress derivatives.

The paper is organized as follow. Section 2 is devoted to the optimization framework used through the paper. The basics of the level set description of the geometry and the XFEM are recalled. Then, the procedure to evaluate the stress fields is explained. The homogenization method is briefly exposed in Section 3 . The attention is drawn to the modification in the numerical procedure due to the use of XFEM. The discrete analytical approach developed to perform the sensitivity analysis is recalled in Section 4 and the computation of the stress derivatives is detailed. Finally, Section 5 illustrates the developed methodology on 2D microstructural designs: finding the optimal shapes and locations of a single or multiple inclusions in a microstructure under various loadings.

\section{Optimization framework}

The objective of this work is to design periodic microstructures minimizing their local stress field. Two phase microstructural design problems in two dimensions are considered. A general description of the problem is given in Fig.(2). Two materials are distributed over a given RVE, $\Omega$. An interface $\Gamma_{A B}$ defines the nonoverlapping subdomains $\Omega_{A}$ and $\Omega_{B}$ filled with material $A$ and $B$ respectively. Periodic boundary conditions are imposed on the RVE as periodic materials are considered. The RVE is submitted to some equivalent loads deduced from prescribed macroscopic strain fields.

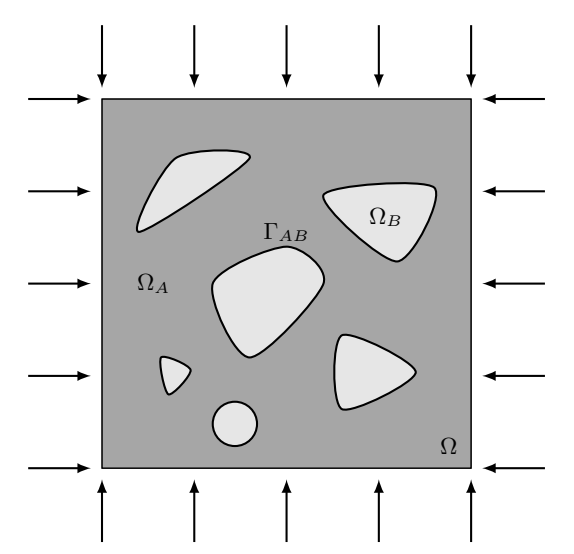

Fig. 2 Description of the microstructural optimization problem.
A bound on the volume $V$ of material phase $A$ or $B$ is prescribed to avoid trivial solutions. Some extra constraints $g_{j}$ with $j=1, \ldots, m$ can also be imposed.

The resulting general optimization problem is formulated as follow:

$$
\begin{array}{ll}
\min _{\mathbf{s}} & \max _{e=1, \ldots, n_{e}}\left\{\sigma_{e}^{\mathrm{VM}}(\mathbf{s})\right\} \\
\text { s.t. } & V(\mathbf{s})-V_{\max }=0 \\
& g_{j}(\mathbf{s}) \leq 0, \quad j=1, \ldots, m \\
& \underline{s}_{i} \leq s_{i} \leq \bar{s}_{i}, \quad i=1, \ldots, n
\end{array}
$$

where $\mathbf{s}$ is a vector collecting the $n$ optimization variables, $\sigma_{e}^{\mathrm{VM}}$ is the Von Mises stress associated to the $e^{t h}$ element of the mesh.

This problem can be reformulated so as to treat the minimization of the maximum Von Mises stress as several stress constraints:

$\min z$

$$
\begin{array}{ll}
\text { s.t. } & \sigma_{e}^{V M}(\mathbf{s}) \leq z, \quad e=1, \ldots, n_{e} \\
& V(\mathbf{s})-V_{\max }=0 \\
& g_{j}(\mathbf{s}) \leq 0, \quad j=1, \ldots, m \\
& \underline{s}_{i} \leq s_{i} \leq \bar{s}_{i}, \quad i=1, \ldots, n \\
& z \geq 0
\end{array}
$$

The optimization problem, proposed in this work, does not fall into the category of inverse homogenization problems. In fact, the homogenization procedure, as exposed in Section 3, is only exploited to impose periodic boundary conditions on the RVE and to evaluate equivalent loads to some prescribed macroscopic strain fields. The full procedure allowing the extraction of the effective properties of a given RVE, is only used as a post-processing operation on the optimization results.

\subsection{Level set description of the geometry}

In 1988, Osher and Sethian [32] introduced the level set method to track moving interfaces efficiently. The method allows the representation of interfaces or discontinuities resorting to an implicit function $\phi$ of dimension $n+1$, while working in a $n$ dimensional space. An iso-level of this function, generally the iso-zero one, is chosen to draw the interface or discontinuity and thus separates the domain between the different materials in presence. The function $\phi$, known as the level set function, can be given as a function of the spatial coordinates $\mathbf{x}$ and some design parameters $\mathbf{s}$ :

$\left\{\begin{array}{l}\phi(\mathbf{x}, \mathbf{s})>0, \forall \mathbf{x} \in \Omega_{A} \\ \phi(\mathbf{x}, \mathbf{s})=0, \forall \mathbf{x} \in \Gamma_{A B} . \\ \phi(\mathbf{x}, \mathbf{s})<0, \forall \mathbf{x} \in \Omega_{B}\end{array}\right.$ 
where $\Omega_{A}$ is the subdomain filled with material A, $\Omega_{B}$ the subdomain filled with material $\mathrm{B}$ and $\Gamma_{A B}$ the interface between the materials A and B.

Implementing FEM on discrete meshes, the level set function $\phi$ is represented by its nodal values $\phi_{i}$ interpolated using classical finite element shape functions $N_{i}(\mathbf{x})$ :

$\phi^{h}(\mathbf{x}, \mathbf{s})=\sum_{i} N_{i}(\mathbf{x}) \phi_{i}$.

In this paper, level set functions are parametrized using a single or a combination of parametrized geometric shapes, as circles, ellipses or superellipses. Instead of evolving the level set function by solving HamiltonJacobi type equations as in Allaire et al. [1] or Wang et al. [50], the level set function is here expressed as a function of the design parameters $\mathbf{s}$ and is modified through mathematical programming algorithms. For more details, the interested reader can refer to van Dijk et al. [11].

\subsection{Extended finite element method}

The XFEM was originally developed by Moës et al. [27] to model crack propagation without remeshing. The method allows representing singular or discontinuous behaviors within a mesh element by adding specific shape functions to classical finite element approximation fields:

$\mathbf{u}^{h}(\mathbf{x})=\sum_{i \in I} N_{i}(\mathbf{x}) u_{i}+\sum_{i \in I^{\star}} N_{i}^{\star}(\mathbf{x}) a_{i}$,

where $I$ is the set of all the mesh nodes, $N_{i}(\mathbf{x})$ are the standard finite element shape functions, $u_{i}$ are the degrees of freedom (dof) associated to the standard shape functions $N_{i}(\mathbf{x}), I^{\star}$ is the set of enriched nodes, $N_{i}^{\star}(\mathbf{x})$ are the enriched shape functions and $a_{i}$ are the additional unknowns related to the enrichment.

The desired enrichment is introduced in the approximations resorting to an enrichment function $\psi$. For a particular application, the choice of the enrichment function $\psi$ depends on the singularities or discontinuities that are to be taken into account. In this work, we focus on interfaces between two materials $A$ and $B$ characterized by a continuous displacement field, but a discontinuous strain field. A commonly used enrichment function to represent material interfaces is the ridge function developed by Moës [26] and given as:

$\psi(\mathbf{x})=\sum_{i} N_{i}(\mathbf{x})\left|\phi_{i}\right|-\left|\sum_{i} N_{i}(\mathbf{x}) \phi_{i}\right|$.

The additional enriched shape functions $N_{i}^{\star}(\mathbf{x})$ are then built by multiplying the classical finite element shape functions $N_{i}(\mathbf{x})$ and the enrichment function $\psi(\mathbf{x})$ :

$N_{i}^{\star}(\mathbf{x})=N_{i}(\mathbf{x}) \psi(\mathbf{x})$.
Since enriched elements are filled with two different materials and present a discontinuity in their strain field, they have to be treated with special care. The different techniques implemented to perform the subdivision and the integration on enriched elements are briefly recalled hereunder.

\subsubsection{XFEM subdivision}

The subdivision of the enriched elements is performed taking advantage of the level set description of the geometry. The level set function $\phi$ is approximated by a straight line for the sake of simplicity. The intersections $\left(\xi^{\star}, \eta^{\star}\right)$ of the iso-zero level curve and the elements edges are computed, in the $2 \mathrm{D}$ case, from the nodal coordinates $\left(\xi_{1}, \eta_{1}\right)$ and $\left(\xi_{2}, \eta_{2}\right)$ and the nodal level set values $\phi_{1}$ and $\phi_{2}$, as shown in Fig.(3):

$$
\begin{aligned}
& \xi^{\star}=t \xi_{2}+(1-t) \xi_{1}, \\
& \eta^{\star}=t \eta_{2}+(1-t) \eta_{1},
\end{aligned} \quad \text { with } \quad t=\frac{\left|\phi_{1}\right|}{\left|\phi_{1}\right|+\left|\phi_{2}\right|} .
$$

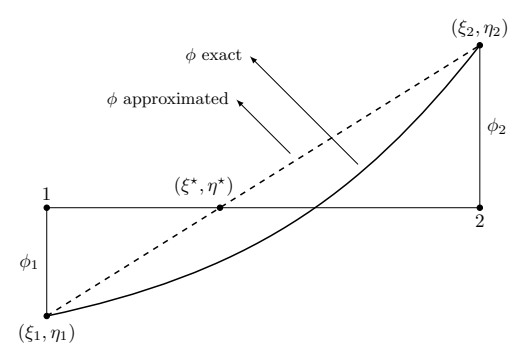

Fig. 3 Subdivision of the enriched elements: intersections of the iso-zero level set and the edge of an element in 2D.

\subsubsection{XFEM integration}

To capture the discontinuous strain behavior through the enriched elements, the integration can no longer be performed using a classical Gauss quadrature. To obtain accurate results, an integration background mesh is introduced on the enriched elements. As illustrated in Figure 4, Gauss points are brought back from a second parametric space $(m, l)$ to the first one $(\xi, \eta)$ where the classical Gauss quadrature integration is carried out on each subpart of the parent element independently. In particular, this procedure is applied to compute the stiffness matrix $\mathbf{K}$. The elemental stiffness matrix is 


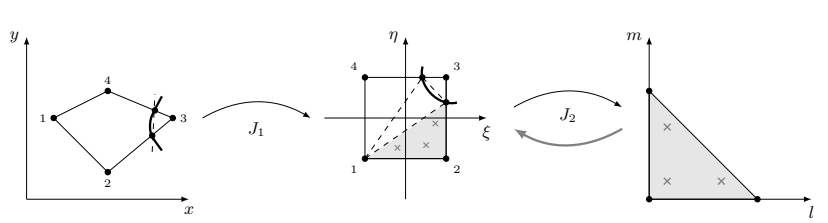

Fig. 4 Integration procedure for enriched elements: the element is mapped to a parent reference element using a first mapping J1. The subdomains of this element are mapped to a second reference space resorting to a set of mappings J2. Gauss points are brought back from the second to the first reference space, where classical Gauss quadrature is performed.

evaluated by adding the contribution of each Gauss point:

$$
\begin{aligned}
\mathbf{K}_{e} & =\int_{\Omega_{e}}\left[\begin{array}{l}
\mathbf{B}_{u}^{T} \\
\mathbf{B}_{a}^{T}
\end{array}\right] \mathbf{H}\left[\begin{array}{ll}
\mathbf{B}_{u} & \mathbf{B}_{a}
\end{array}\right] d \Omega_{e}, \\
& \simeq \sum_{g p} w_{g p}\left[\begin{array}{l}
\mathbf{B}_{u}^{T} \\
\mathbf{B}_{a}^{T}
\end{array}\right]_{g p} \mathbf{H}_{g p}\left[\begin{array}{ll}
\mathbf{B}_{u} & \mathbf{B}_{a}
\end{array}\right]_{g p}\left|\mathbf{J}_{1}\right|_{g p}\left|\mathbf{J}_{2}\right|_{g p},
\end{aligned}
$$

where $\Omega_{e}$ is the element domain, $\sum_{g p}$ is the sum over all the element Gauss points, $\mathbf{B}_{u}$ is the part of the $\mathbf{B}$ matrix of the derived shape functions related to the classical dof $u_{i}, \mathbf{B}_{a}$ is the part of the $\mathbf{B}$ matrix of the derived shape functions related to the additional dof $a_{i}, \mathbf{H}$ is the Hooke's matrix, $\left|\mathbf{J}_{1}\right|$ is the determinant of the first Jacobian mapping, $\left|\mathbf{J}_{2}\right|$ is the determinant of the second Jacobian mapping, $w_{g p}$ are the weights associated to Gauss points.

\subsubsection{Evaluation of the stress}

As the objective of this work is to take into account the stress fields within the microstructures, the procedure to evaluate the stress values is detailed hereunder. For each Gauss point, the stress tensor is evaluated through:

$\boldsymbol{\sigma}_{g p}=\mathbf{H}_{g p} \mathbf{B}_{g p} \mathbf{u}_{e}$,

where $\mathbf{u}_{e}$ is a vector containing the dof of the element where the considered Gauss point is located.

The Gauss points stress tensors are then arranged as a mean by element or by node. If an element is crossed by the interface and is filled with two different materials, the stress tensors at the Gauss points are treated differently. Working with a mean by element strategy, a mean stress tensors is evaluated for each subpart of the parent element. Working with a mean by node strategy, additional stress tensors are evaluated at the intersections between the interface and the element edges. In this work, the mean by element strategy is adopted as it is straightforward and provides rather accurate results.

As indicated in Van Miegroet and Duysinx [46], on an enriched element, as the area dedicated to one ma- terial becomes smaller, the stress value on this vanishing area subpart can be highly overestimated. Several strategies of pre- and post-processing are proposed in the literature to overcome these difficulties and obtain a smooth stress field. Among those, one can cite:

- moving the node on the interface to remove the small subpart and the corresponding overestimated stress. However, this pre-processing procedure implies working with a moving mesh, which is contradictory to the reason invoked to use the XFEM, i.e. working on fixed meshes.

- moving the interface to the closest node to remove the small subpart and the corresponding overestimated stress. This pre-processing strategy, known as fit-to-vertex, was proposed by Moës et al. [28].

- evaluating nodal stresses using recovery techniques based on finite element error estimation. These postprocessing stress recovery procedures are widely used, as they allow to rebuild more accurate nodal values of the stress from the finite element solution. A survey of different techniques is given by Krizek and Neittaanmaki [21]. For example, Zienkenwick and Zhu [53] proposed a superconvergent patch recovery technique that uses a polynomial function to describe the stress on an element patch surrounding the treated node. So far, these techniques were mainly applied with XFEM to recover a consistent stress field around cracks as in Bordas and Duflot [8] and in Ródenas et al. [34].

- smoothing the stress on small element subparts by an area weighted sum over the element and its neighbors, as proposed by Van Miegroet and Duysinx in [46]. This post-processing strategy requires to build the mesh topology so that each element is associated to its neighbors. Nevertheless, as the mesh is fixed, only one evaluation of the mesh topology is necessary.

Although not being the most accurate approach, the latter strategy is simple and straightforward. The approach can not ensure that no overestimated stress value will appear but it provides rather good results in many cases as shown by Van Miegroet [45]. It is therefore adopted in this article and detailed hereunder.

The smoothed stress tensor $\sigma^{S}$ associated to a given subelement is given as:

$\sigma^{S}=\frac{\sum_{e \in N} A_{e} \sigma_{e}}{\sum_{e \in N} A_{e}}$,

where $N$ is a set of the treated subelement and its neighbors as shown in Fig.(5), $A_{e}$ is the area of element $e$, $\sigma_{e}$ is the stress tensor on element $e$. 


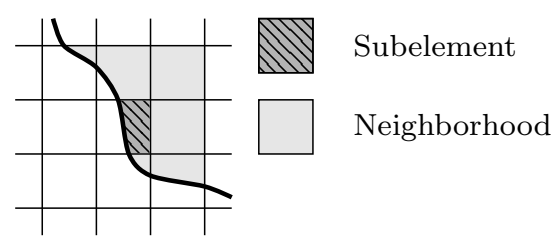

Fig. 5 Smoothing the stress field while working with XFEM: neighborhood of a given subelement.

\section{Homogenization}

As the layout of materials at the microscopic level can be extremely complex, it is not possible to study these as a whole. To overcome this difficulty, the theory of homogenization was developed in the 1970s and allows extracting effective homogenized properties at the macroscopic level. Initial works on homogenization were provided by Bensoussan et al. [7] and Sanchez-Palencia [35]. They studied periodic materials built from the repetition of RVEs in two or three dimensions.

In this paper, we focus on orthotropic macroscopic materials. Their constituent materials are considered as linear elastic and isotropic. Therefore, the constitutive equations, at the microscopic scale, are given as follow:

$\boldsymbol{\sigma}=\mathbf{H} \epsilon$,

where $\boldsymbol{\sigma}$ is the stress vector, $\boldsymbol{\epsilon}$ the strain vector and $\mathbf{H}$ the Hooke's matrix.

The homogenized properties of a periodic material can be deduced from its microstructure as the strain energy evaluated at the macroscopic level in terms of macroscopic strains should be equal to the strain energy calculated from the microscopic strain field. Working in $2 \mathrm{D}$, the homogenized coefficients $H_{i j}^{H}$ are obtained applying three independent unit strain fields $\boldsymbol{\epsilon}_{0}^{i}$ :

$$
\begin{gathered}
H_{i j}^{H}=\frac{1}{Y} \int_{Y}\left(\boldsymbol{\epsilon}_{0}^{i}-\boldsymbol{\epsilon}^{i}\right)^{T} \mathbf{H}\left(\boldsymbol{\epsilon}_{0}^{j}-\boldsymbol{\epsilon}^{j}\right)^{T} d Y, \\
i, j=1, \ldots, 3
\end{gathered}
$$

where $Y$ is the volume of the RVE, $\boldsymbol{\epsilon}_{0}^{i}$ are the three prescribed macroscopic strain field (in $2 \mathrm{D} \epsilon_{0}^{1}=\left[\begin{array}{lll}1 & 0 & 0\end{array}\right]^{T}$, $\boldsymbol{\epsilon}_{0}^{2}=\left[\begin{array}{lll}0 & 1 & 0\end{array}\right]^{T}$ and $\left.\boldsymbol{\epsilon}_{0}^{3}=\left[\begin{array}{lll}0 & 0 & 1\end{array}\right]^{T}\right), \boldsymbol{\epsilon}^{i}$ are the local varying strain fields in the RVE when the latter is submitted to the unit strain field $\boldsymbol{\epsilon}_{0}^{i}$.

The chosen XFEM-level set framework is used to extract the equivalent macroscopic properties from the microstructures, performing numerical homogenization. The procedure followed in this paper, inspired by works by Mlejnek and Schirrmacher [25] or more recent similar works by Andreassen and Andreasen [3], is adapted to the particular framework.
First, the periodic boundary conditions are applied through the elimination of redundant dofs. Two corresponding nodes are associated and share the same dofs, as described in Fig.(6).

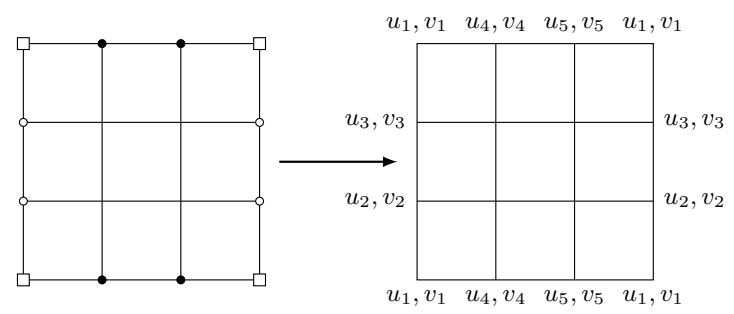

Fig. 6 Applying periodic boundary conditions on 2D square RVE via the elimination of redundant dofs. The nodes are associated to their corresponding ones: the four corners of the cell are associated and each left (resp. bottom) white (resp. black) node is paired with its corresponding right (resp. top) one. Then, associated nodes share the same dofs.

Then, the displacement field $\mathbf{u}^{i}$, induced in the RVE by the imposition of the unit strain field $\boldsymbol{\epsilon}_{0}^{i}$, has to be evaluated. It is done by solving the following equations:

$\mathbf{K} \mathbf{u}^{i}=\mathbf{f}_{0}^{i}, \quad i=1, \ldots, 3$,

where $\mathbf{K}$ is the stiffness matrix of the RVE, $\mathbf{u}^{i}$ is the displacement field induced by prescribing the unit strain field $\boldsymbol{\epsilon}_{0}^{i}$ on the RVE and $\mathbf{f}_{0}^{i}$ is the load vector equivalent to the application of the unit strain field $\epsilon_{0}^{i}$ on the RVE.

In an XFEM framework, as explained in section 2.2.3, the classical Gauss quadrature can no longer be applied. The stiffness matrix is then evaluated as in Eq.(9). The equivalent load vectors $\mathbf{f}_{0}^{i}$ are evaluated as:

$$
\begin{aligned}
\mathbf{f}_{0}^{i}= & \int_{Y} \mathbf{B}^{T} \mathbf{H} \boldsymbol{\epsilon}_{0}^{i} d Y \\
\simeq & \sum_{g p} w_{g p} \mathbf{B}_{g p}^{T} \mathbf{H}_{g p} \boldsymbol{\epsilon}_{0}^{i}\left|\mathbf{J}_{1}\right|_{g p}\left|\mathbf{J}_{2}\right|_{g p}, \\
& \quad i=1, \ldots, 3 .
\end{aligned}
$$

Finally, the homogenized coefficients $H_{i j}^{H}$ are evaluated through:

$$
\begin{aligned}
H_{i j}^{H}= & \frac{1}{Y} \int_{Y}\left(\mathbf{u}_{0}^{i}-\mathbf{u}^{i}\right)^{T} \mathbf{B}^{T} \mathbf{H} \mathbf{B}\left(\mathbf{u}_{0}^{j}-\mathbf{u}^{j}\right) d Y \\
= & \frac{1}{Y}\left(\mathbf{u}_{0}^{i}-\mathbf{u}^{i}\right)^{T} \mathbf{K}\left(\mathbf{u}_{0}^{j}-\mathbf{u}^{j}\right) \\
& i, j=1, \ldots, 3
\end{aligned}
$$

where $\mathbf{u}_{0}^{i}$ is the displacement field equivalent to the prescribed unit strain field $\boldsymbol{\epsilon}_{0}^{i}$ and $\mathbf{u}^{i}$ is the displacement field induced by the imposition of the unit strain field $\epsilon_{0}^{i}$ on the RVE. 


\section{Sensitivity analysis}

This section focuses on the approach developed to perform the sensitivity analysis as proposed in Noël et al. [31]. Working within an XFEM-level set framework, performing the sensitivity analysis by finite difference or a semi-analytical approach can lead to problems and inaccuracies in the derivatives values. In fact, the perturbation of the interface or discontinuity can imply a change in the status of the elements from enriched to not enriched and vice versa, resulting in a modification of the approximation/discretization fields used since new shape functions are introduced or retrieved. Therefore, as suggested in [31], an analytical approach to the sensitivity analysis can be successfully applied.

\subsection{Sensitivity and homogenization}

Continuing along previous works, the developed analytical sensitivity analysis, adapted to the XFEM-level set framework, is used to evaluate the requested derivatives. Some important features for further developments are recalled.

Starting from the discretized equations of the linear elasticity $\mathbf{K u}=\mathbf{f}$, where $\mathbf{K}$ is the stiffness matrix, $\mathbf{u}$ the displacements vector and $\mathbf{f}$ the vector of the external forces, the equations are derived with respect to a design parameter $s$, it results:

$\frac{\partial \mathbf{K}}{\partial s} \mathbf{u}+\mathbf{K} \frac{d \mathbf{u}}{d s}=\frac{d \mathbf{f}}{d s} \Leftrightarrow \frac{d \mathbf{u}}{d s}=-\mathbf{K}^{-1}\left[\frac{\partial \mathbf{K}}{\partial s} \mathbf{u}-\frac{d \mathbf{f}}{d s}\right]$

Thus, knowing the derivatives of the stiffness matrix $\mathbf{K}$ and of the vector of the external forces $\mathbf{f}$, the sensitivity of the displacements vector $\mathbf{u}$ can be readily evaluated.

The stiffness matrix derivative is evaluated starting from its discretized equation and deriving it with respect to a particular design parameter $s$. Working with an XFEM-level set framework, one gets:

$$
\begin{aligned}
\frac{\partial \mathbf{K}}{\partial s} & =\sum_{g p} w \frac{\partial \mathbf{B}^{T}}{\partial s} \mathbf{H} \mathbf{B}\left|\mathbf{J}_{1}\right|\left|\mathbf{J}_{2}\right| \\
& +\sum_{g p} w \mathbf{B}^{T} \mathbf{H} \frac{\partial \mathbf{B}}{\partial s}\left|\mathbf{J}_{1}\right|\left|\mathbf{J}_{2}\right| \\
& +\sum_{g p} w \mathbf{B}^{T} \mathbf{H} \mathbf{B}\left|\mathbf{J}_{1}\right| \frac{\partial\left|\mathbf{J}_{2}\right|}{\partial s} .
\end{aligned}
$$

The equivalent load vector $\mathbf{f}_{0}$ is dependent on the design parameters and its derivative is obtained following the same procedure. Its discretized expression, given in Eq.(15) is derived with respect to a particular design parameter $s$ :

$$
\begin{aligned}
\frac{\partial \mathbf{f}_{0}}{\partial s}= & \sum_{g p} w \frac{\partial \mathbf{B}^{T}}{\partial s} \mathbf{H} \boldsymbol{\epsilon}_{0}\left|\mathbf{J}_{1}\right|\left|\mathbf{J}_{2}\right| \\
+ & \sum_{g p} w \mathbf{B}^{T} \mathbf{H} \boldsymbol{\epsilon}_{0}\left|\mathbf{J}_{1}\right| \frac{\partial\left|\mathbf{J}_{2}\right|}{\partial s} \\
& i=1, \ldots, 3
\end{aligned}
$$

The derivative of the stiffness matrix, as well as the derivative of the equivalent load vector, require to evaluate the derivatives of the $\mathbf{B}$ matrix and of the determinant of the second Jacobian mapping $\mathbf{J}_{2}$ with respect to the design parameter $s$.

The $\mathbf{B}$ matrix is made of two parts: $\mathbf{B}_{u}$, related to the classical dof $u_{i}$, contains the derivatives of the classical shape function $N_{i}(\mathbf{x})$ with respect to $(\xi, \eta)$ the coordinates of the first reference space and $\mathbf{B}_{a}$, related to the enrichment additional dof $a_{i}$, contains the derivatives of the enriched shape functions $N_{i}^{\star}(\mathbf{x})$ with respect to $(\xi, \eta)$ the coordinates of the first reference space.

The derivative of the $\mathbf{B}_{u}$ matrix with respect to a design parameter $s$ is built using the following terms:

$\frac{\partial}{\partial s}\left[\begin{array}{c}\frac{\partial N_{i}}{\partial x} \\ \frac{\partial N_{i}}{\partial y}\end{array}\right]=\mathbf{J}_{1}^{-1}\left[\begin{array}{c}\frac{\partial^{2} N_{i}}{\partial \xi^{2}} \frac{\partial \xi}{\partial s}+\frac{\partial^{2} N_{i}}{\partial \xi \partial \eta} \frac{\partial \eta}{\partial s} \\ \frac{\partial^{2} N_{i}}{\partial \eta \partial \xi} \frac{\partial \xi}{\partial s}+\frac{\partial^{2} N_{i}}{\partial \eta^{2}} \frac{\partial \eta}{\partial s}\end{array}\right]$.

The derivative of the $\mathbf{B}_{a}$ matrix with respect to a design parameter $s$ is built using the following terms:

$$
\begin{aligned}
\frac{\partial}{\partial s}\left[\begin{array}{c}
\frac{\partial N_{i}^{\star}}{\partial x} \\
\frac{\partial N_{i}^{\star}}{\partial y}
\end{array}\right]=\mathbf{J}_{1}^{-1}\left(\left[\begin{array}{c}
\frac{\partial^{2} N_{i}}{\partial \xi^{2}} \frac{\partial \xi}{\partial s}+\frac{\partial^{2} N_{i}}{\partial \xi \partial \eta} \frac{\partial \eta}{\partial s} \\
\frac{\partial^{2} N_{i}}{\partial \eta^{2}} \frac{\partial \eta}{\partial s}+\frac{\partial^{2} N_{i}}{\partial \eta \partial \xi} \frac{\partial \xi}{\partial s}
\end{array}\right] \psi\right. \\
+\left[\begin{array}{c}
\frac{\partial N_{i}}{\partial \xi} \\
\frac{\partial N_{i}}{\partial \eta}
\end{array}\right] \frac{\partial \psi}{\partial s}+\frac{\partial}{\partial s}\left[\begin{array}{c}
\frac{\partial \psi}{\partial \xi} \\
\frac{\partial \psi}{\partial \eta}
\end{array}\right] N_{i} \\
\left.+\left[\begin{array}{c}
\frac{\partial \psi}{\partial \xi} \\
\frac{\partial \psi}{\partial \eta}
\end{array}\right]\left(\frac{\partial N_{i}}{\partial \xi} \frac{\partial \xi}{\partial s}+\frac{\partial N_{i}}{\partial \eta} \frac{\partial \eta}{\partial s}\right)\right)
\end{aligned}
$$

The derivative of the determinant of the second Jacobian mapping $\mathbf{J}_{2}$ with respect to a design parameter $s$ is given as:

$$
\begin{aligned}
\frac{\partial\left|\mathbf{J}_{2}\right|}{\partial s}=\left(\sum_{i}\right. & \left.\frac{\partial N_{i}}{\partial l} \frac{\partial \xi_{i}}{\partial s}\right)\left(\sum_{i} \frac{\partial N_{i}}{\partial m} \eta_{i}\right) \\
& +\left(\sum_{i} \frac{\partial N_{i}}{\partial l} \xi_{i}\right)\left(\sum_{i} \frac{\partial N_{i}}{\partial m} \frac{\partial \eta_{i}}{\partial s}\right) \\
& -\left(\sum_{i} \frac{\partial N_{i}}{\partial m} \frac{\partial \xi_{i}}{\partial s}\right)\left(\sum_{i} \frac{\partial N_{i}}{\partial l} \eta_{i}\right) \\
& -\left(\sum_{i} \frac{\partial N_{i}}{\partial m} \xi_{i}\right)\left(\sum_{i} \frac{\partial N_{i}}{\partial l} \frac{\partial \eta_{i}}{\partial s}\right) .
\end{aligned}
$$

As in classical shape optimization, the evaluation of these derivatives requires the definition and the computation of a velocity field appearing in the equations 
through the following terms: $d \xi / d s, d \eta / d s$. In classical shape optimization, the velocity field describes the perturbation of the nodal positions with respect to modifications of the boundaries and has to be evaluated over the entire design domain. Resorting to an XFEMlevel set framework, a fixed mesh is used and the velocity field describes the material flow through the intersected elements. Therefore, its evaluation is advantageously limited to enriched elements. All the details about the derivatives involved in these computations are explained in [31] and the interested reader can refer to the latter paper to find any additional information.

\subsection{Sensitivity of the stresses}

The developed analytical sensitivity analysis is also used to evaluate the derivatives of the stress field.

Starting from Eq.(10) and deriving it with respect to a design parameter $s$, one gets:

$\frac{d \boldsymbol{\sigma}_{g p}}{d s}=\mathbf{H}_{g p} \frac{\partial \mathbf{B}_{g p}}{\partial s} \mathbf{u}_{e}+\mathbf{H}_{g p} \mathbf{B}_{g p} \frac{d \mathbf{u}_{e}}{d s}$

which only requires the derivatives of the $\mathbf{B}$ matrix given in Eq.(20), Eq.(21) and of the displacements vector given by Eq.(17).

As pointed out, these derivatives are evaluated directly from the values extracted during the analysis and a very small subpart in an element crossed by the interface can be associated with a highly overestimated stress derivative. The latter can lead to convergence problems through the optimization process. Therefore, the same pre- and post-processing strategies mentioned in 2.2.3 can be applied. By proceeding this way, smoothed stress derivative values can be recovered.

\section{Applications}

The developed approach is illustrated on 2D examples. It is first validated on a classical shape optimization problem: a single inclusion in a microstructure undergoing different loadings. In particular, the Vigdergauz microstructures are investigated as they can achieve extremal elastic behavior while exhibiting a rather simple geometry. Later on, the case of microstructures reinforced by several stiff inclusions is investigated to illustrate that the approach is able to generate more complex microstructural designs.

Optimization problems are formulated as detailed in Section 2 and solved resorting to mathematical programming schemes, as the Method of Moving Asymptotes (MMA) of Svanberg [42].
5.1 Single inclusion microstructures under hydrostatic and shear loadings

As a first example, single inclusion microstructures are investigated. The design problem aims at finding the optimal shape of a hole minimizing the surrounding Von Mises stresses in a square RVE. The material behaviors are considered linear elastic and isotropic. Working with given microscopic material properties and assuming perfect bonding in the case of two-phase composite, the shape of the hole is the only parameter to determine the stress field in the microstructure. Therefore, this problem is well-suited to evaluate the accuracy and the efficiency of the developed approach. Two loading cases are considered: a hydrostatic and a pure shear loading.

\subsubsection{Hydrostatic loading}

First, a hydrostatic loading is considered: $\boldsymbol{\epsilon}_{\mathrm{hydro}}=[-1-$ $10]^{T}$. This hydrostatic loading was used as trial loading while seeking to maximize the effective bulk modulus of the microstructure and to saturate the corresponding Hashin-Shtrikman bounds [17]. Under hydrostatic loading and satisfying the traction-free condition on the hole, Vigdergauz [48] was able to identify the optimal contour of the hole minimizing the strain energy analytically. A family of elliptical shapes, evolving from circles for low hole volume to squares with rounded corners for higher hole volume, are maximizing the effective bulk moduli of the material. These shapes also satisfy the equi-stress principle, i.e. the non zero stresses along the hole are uniform. Later on, Grabovsky and Kohn [14] demonstrated that minimizing the strain energy was equivalent to minimizing the stress concentration in the case of a single inclusion microstructure undergoing a single macroscopic load case.

In the following numerical investigations, two different objectives are then pursued and compared: minimizing the strain energy $C$ and minimizing the local Von Mises stresses $\sigma^{\mathrm{VM}}$. In both cases, the optimal shape of a hole in a square RVE is sought. The hole is represented by a superellipse with a prescribed target volume $V_{\max }$. The superelliptical hole is characterized by two geometric features used as design parameters: $a$, its semi-axis length and $\xi$, its exponent. The hole is modeled by a very soft material, i.e. $E_{\text {solid }} / E_{\text {soft }}=10^{6}$. Proceeding this way, the chosen XFEM approximation for material interface can be applied directly without constraining the dofs within the void regions. Both materials have an isotropic and linear elastic behavior. A 
plane stress state is assumed. The optimization problems to solve are formulated as follow:

\begin{tabular}{c|c} 
Strain energy: & Von Mises stress: \\
\hline $\min _{\mathbf{s}} C$ & $\min _{\mathbf{s}} \max _{e=1, \ldots, n_{e}}\left\{\sigma_{e}^{\mathrm{VM}}\right\}$ \\
s.t. $V_{\text {hole }}=V_{\max }$ & s.t. $V_{\text {hole }}=V_{\max }$ \\
$\underline{\mathbf{s}} \leq \mathbf{s} \leq \overline{\mathbf{s}}$ & $\underline{\mathbf{s}} \leq \mathbf{s} \leq \overline{\mathbf{s}}$
\end{tabular}

The stress design problem is reformulated as detailed in Eq.(2). The stopping criteria are the relative variations of the design parameters that have to be smaller than $10^{-3}$. The initial configuration, the imposed boundary conditions and the mesh are presented in Fig.(7). All the parameters used through the optimization process are given in Table 1.

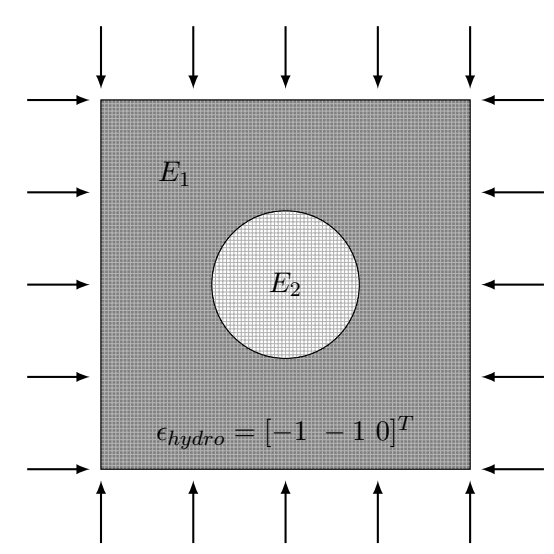

Fig. 7 Single inclusion microstructure under hydrostatic loading: description of the problem.

Table 1 Single inclusion microstructure under hydrostatic loading: parameters.

\begin{tabular}{lll}
\hline Dimensions & {$[\mathrm{m}]$} & $c=10, t=1$ \\
Elastic moduli & {$\left[\mathrm{N} / \mathrm{m}^{2}\right]$} & $E_{1}=1, E_{2}=10^{-6}$ \\
Poisson's ratio & {$[-]$} & $\nu_{1}=\nu_{2}=0.3$ \\
Hydrostatic loading & {$[-]$} & $\epsilon_{\mathrm{hydro}}=\left[\begin{array}{ll}-1-1 & 0\end{array}\right]^{T}$ \\
Bound on $V_{\text {hole }}$ & {$\left[\mathrm{m}^{3}\right]$} & $V_{\max }=20,40,60,80$ \\
Bounds on design variables & {$[\mathrm{m}]$} & $0 \leq a \leq 4.9$ \\
& {$[-]$} & $1 \leq \xi \leq 20$ \\
Level set function & & $\phi(\mathbf{x}, \mathbf{s})=\left(\frac{x}{a}\right)^{\xi}+\left(\frac{y}{a}\right)^{\xi}-1$ \\
Gauss points per subelement & $n_{g p}=7$ \\
Convergence criteria & $\Delta a, \Delta \xi \leq 10^{-3}$ \\
Mesh & $100 \times 100$ bilinear Q4 \\
\hline
\end{tabular}

The optimized shapes found for target hole volumes of 20,40,60 and $80 \mathrm{~m}^{3}$ for both objectives are shown in Fig.(8). As expected, both formulations lead to very similar results. Moreover, the obtained results are in good agreement with Vigdergauz numerical results, as presented in [48]. For growing target hole volumes, expected behavior is recovered, i.e. optimized shapes evolve roughly from circular shapes to squares with rounded corners. Although the local Von Mises stresses are minimized instead of the stress concentration, Fig.(9) shows that the stress fields around the holes are rather smooth and that stress concentrations are avoided.

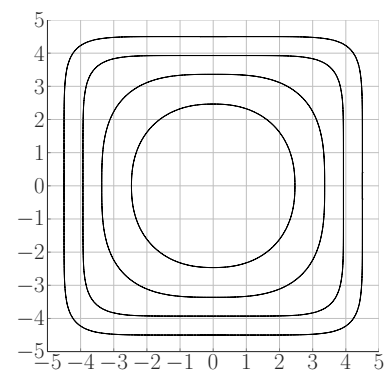

(a) Strain energy.

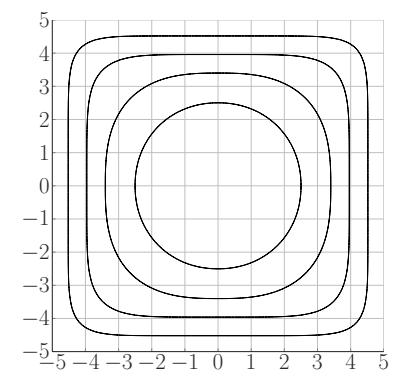

(b) Von Mises stress.
Fig. 8 Single inclusion microstructure under hydrostatic loading: optimized geometries for target volumes of 20,40 , 60 and $80 \mathrm{~m}^{3}$.

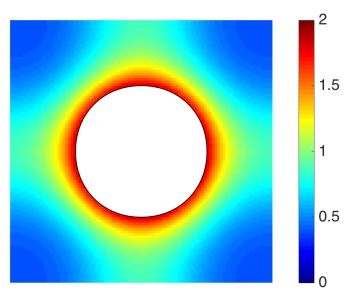

(a) $V_{\text {hole }}=20 \mathrm{~m}^{3}$.

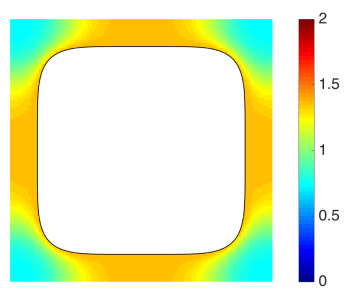

(c) $V_{\text {hole }}=60 \mathrm{~m}^{3}$.

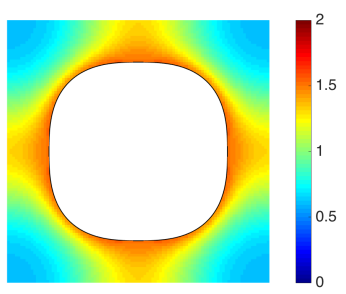

(b) $V_{\text {hole }}=40 \mathrm{~m}^{3}$.

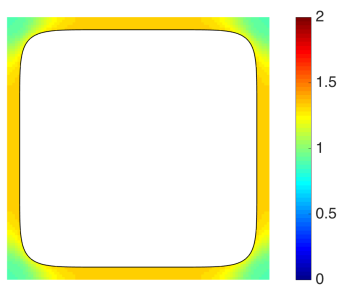

(d) $V_{\text {hole }}=80 \mathrm{~m}^{3}$.
Fig. 9 Single inclusion microstructure under hydrostatic loading: smoothed Von Mises stress fields $\left[\mathrm{N} / \mathrm{m}^{2}\right]$ in the sense of Eq.(11) around the optimized holes for target volumes of $20,40,60$ and $80 \mathrm{~m}^{3}$.

To further validate the numerical results, the effective bulk modulus $\kappa^{H}$ is evaluated for the different hole volumes. They are compared against the HashinShtrikman bounds. As the results are very similar for the strain energy and the stress formulation, only the 
stress designs are considered here. Theoretically, the optimized microstructures should maximize the bulk modulus and saturate the Hashin-Shtrikman upper bound. In plane elasticity, the effective bulk modulus $\kappa^{H}$ can be recollected from the homogenized coefficients $H_{i j}^{H}$ and is given as:

$\kappa^{H}=\frac{E^{H}}{2\left(1-\nu^{H}\right)}$.

For two-phase materials, in the case of plane elasticity and one material being void, the Hashin-Shtrikman bounds are given as a function of the microstructure density $\rho$, the bulk and the shear modulus of the solid phase $\kappa_{\text {solid }}$ and $\mu_{\text {solid }}[44],[5]$ :

$0 \leq \kappa^{H S} \leq \frac{\rho \kappa_{\text {solid }} \mu_{\text {solid }}}{(1-\rho) \kappa_{\text {solid }}+\mu_{\text {solid }}}$.

The comparison is summarized in Fig.(10). It clearly shows that the optimized stress designs are effectively able to saturate the Hashin-Shtrikman upper bound.

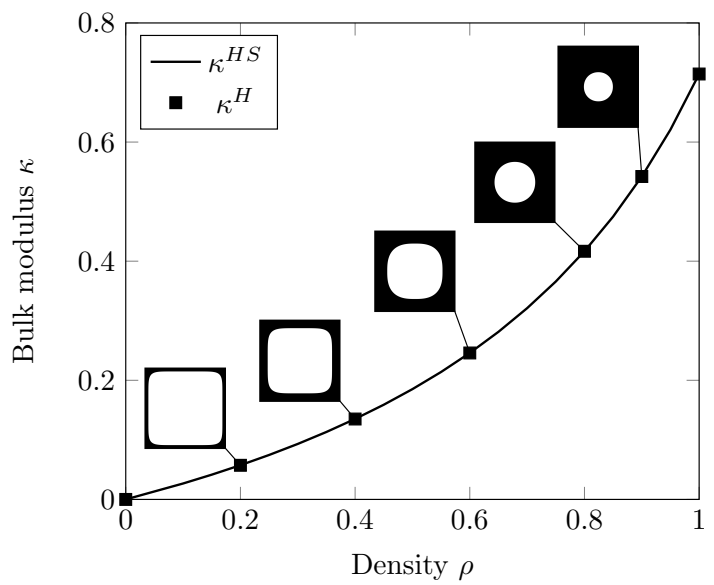

Fig. 10 Single inclusion microstructure under hydrostatic loading: comparison of the bulk modulus obtained numerically $\kappa^{H}$ with the Hashin-Shtrikman bounds $\kappa^{H S}$.

To emphasize the choice of an initial superelliptical shape, the performance of the optimized designs is evaluated in terms of maximal Von Mises stress achieved for various initial shapes. In particular, a circle and an ellipse are considered. For growing hole volumes, the maximum Von Mises stress for each initial shape is presented in Table 2. No results are provided for the circle and the ellipse for a target hole volume of $80 \mathrm{~m}^{3}$ as such a high volume can not be reached while remaining inside the RVE. For low hole volume, the maximum Von Mises stress is very similar regardless of the initial shape. This was expected as the optimal shape is, in this case, close to a circle. For higher hole volume, the maximum Von Mises stress is lower when a superellipse is used as the optimal shape is a square with rounded corners.

Table 2 Single inclusion microstructure under hydrostatic loading: influence of the initial shape on the maximum Von Mises stress achieved.

\begin{tabular}{l|cccc}
$\sigma_{\max }^{\mathrm{VM}}\left[N / m^{2}\right]$ for $\backslash V_{\max }\left[m^{3}\right]$ & 20 & 40 & 60 & 80 \\
\hline Circle & 1.871 & 1.744 & 1.796 & $/$ \\
Ellipse & 1.870 & 1.744 & 1.796 & $/$ \\
Superellipse & 1.823 & 1.550 & 1.374 & 1.326
\end{tabular}

\subsubsection{Shear loading}

In this second case, a pure shear loading is considered: $\boldsymbol{\epsilon}_{\text {shear }}=\left[\begin{array}{lll}-1 & 1 & 0\end{array}\right]^{T}$. This pure shear loading was used as trial loading while seeking to maximize the effective shear modulus of the microstructure and to saturate the corresponding Hashin-Shtrikman bounds. Under pure shear loading, the equi-stress principle is not applicable anymore. Vigdergauz [48] was only able to identify an energy minimizing contour of the hole presenting several angular points. The latter allows the non zero stresses along the hole to change sign while keeping a constant value, i.e. they satisfy the modular equistress principle. These suboptimal shapes correspond to squares with sharp corners and slightly rounded sides.

As for the hydrostatic loading, two objectives are pursued and compared: minimizing the strain energy $C$ and minimizing the local Von Mises stresses $\sigma^{\mathrm{VM}}$. The optimal shape of a hole in a square RVE is sought, but the hole is now represented by the intersection of two superellipses. Six geometric features are used as design parameters: $a_{i}$, the horizontal semi-axis lengths, $b_{i}$ the vertical semi-axis lengths and $\xi_{i}$, the exponents with $i=1,2$. The hole is modeled by a very soft material, i.e. $E_{\text {solid }} / E_{\text {soft }}=10^{6}$. Both materials have an isotropic and linear elastic behavior. A plane stress state is assumed. The two optimization problems to solve are formulated as in Eq.(24). The stress design problem is reformulated as detailed in Eq.(2). The stopping criteria are the relative variations of the design parameters that have to be smaller than $10^{-3}$. The initial configuration, the imposed boundary conditions and the mesh are presented in Fig.(11). All the parameters used through the optimization process are given in Table 3 .

The optimized shapes found for target hole volumes of 20,40,60 and $80 \mathrm{~m}^{3}$ for both objectives are shown in Fig.(12). Anew both formulations in strain energy and stress lead to very similar solutions. The obtained results are in good agreement with Vigdergauz numerical results, as exposed in [48]. Figure 13 shows that the 


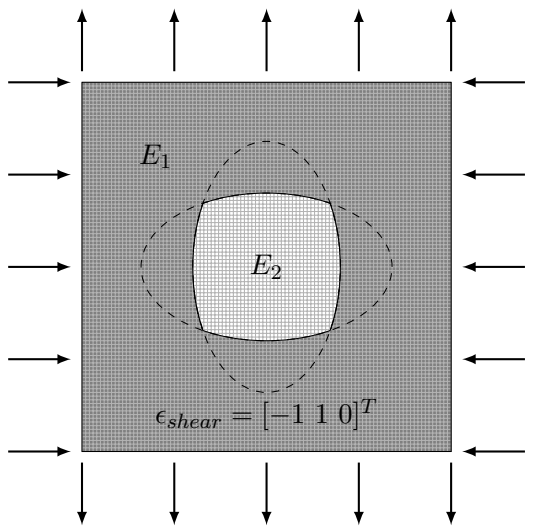

Fig. 11 Single inclusion microstructure under pure shear loading: description of the problem.

Table 3 Single inclusion microstructure under pure shear loading: parameters.

\begin{tabular}{|c|c|c|}
\hline$\overline{\text { Dimensions }}$ & {$[\mathrm{m}]$} & $c=10, t=1$ \\
\hline Elastic moduli & {$\left[N / m^{2}\right]$} & $E_{1}=1, E_{2}=10^{-6}$ \\
\hline Poisson's ratio & {$[-]$} & $\nu_{1}=\nu_{2}=0.3$ \\
\hline Hydrostatic loading & {$[-]$} & $\boldsymbol{\epsilon}_{\text {shear }}=\left[\begin{array}{lll}-1 & 1 & 0\end{array}\right]^{T}$ \\
\hline Bound on $V_{\text {hole }}$ & {$\left[m^{3}\right]$} & $V_{\max }=20,40,60,80$ \\
\hline \multirow[t]{3}{*}{ Bounds on design variables } & {$[m]$} & $0 \leq a_{i} \leq 4.9$ \\
\hline & {$[m]$} & $0 \leq b_{i} \leq 4.9$ \\
\hline & {$[-]$} & $1 \leq \xi_{i} \leq 20$ \\
\hline \multirow[t]{3}{*}{ Level set function } & & $\phi_{1}\left(\mathbf{x}, \mathbf{s}_{1}\right)=\left(\frac{x}{a_{1}}\right)^{\xi_{1}}+\left(\frac{y}{b_{1}}\right)^{\xi_{1}}-1$ \\
\hline & & $\phi_{2}\left(\mathbf{x}, \mathbf{s}_{2}\right)=\left(\frac{x}{a_{2}}\right)^{\xi_{2}}+\left(\frac{y}{b_{2}}\right)^{\xi_{2}}-1$ \\
\hline & & $\phi_{\text {global }}(\mathbf{x}, \mathbf{s})=\phi_{1}\left(\mathbf{x}, \mathbf{s}_{1}\right) \cup \phi_{2}\left(\mathbf{x}, \mathbf{s}_{2}\right)$ \\
\hline Gauss points per subelement & & $n_{g p}=7$ \\
\hline Convergence criteria & & $\Delta a_{i}, \Delta b_{i}, \Delta \xi_{i} \leq 10^{-3}$ \\
\hline Mesh & & $100 \times 100$ bilinear $\mathrm{Q} 4$ \\
\hline
\end{tabular}

Von Mises stress fields for the different target volumes are quite smooth, avoiding stress concentration in the microstructure.

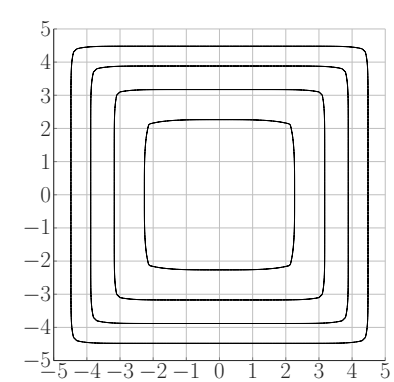

(a) Strain energy.

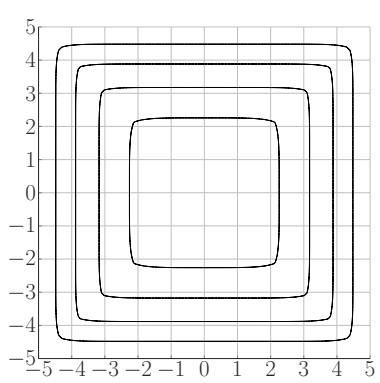

(b) Von Mises stress.
Fig. 12 Single inclusion microstructure under pure shear loading: optimized geometries for target volumes of 20,40, 60 and $80 \mathrm{~m}^{3}$.

As explained previously, these contours are only suboptimal and are unable to saturate the Hashin-Shtrikman bounds on the shear modulus. Nevertheless, the effective shear modulus $\mu^{H}$ is evaluated for the different

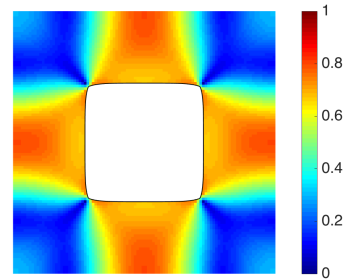

(a) $V_{\text {hole }}=20 \mathrm{~m}^{3}$.

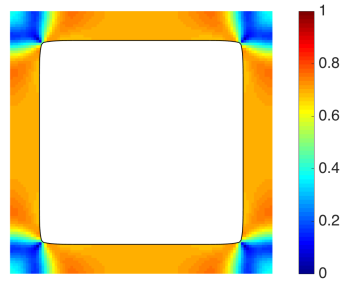

(c) $V_{\text {hole }}=60 \mathrm{~m}^{3}$.

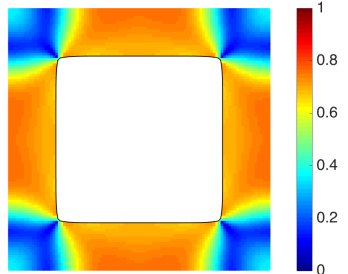

(b) $V_{\text {hole }}=40 \mathrm{~m}^{3}$.

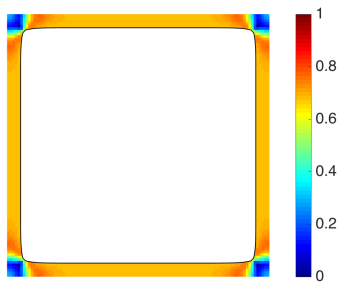

(d) $V_{\text {hole }}=80 \mathrm{~m}^{3}$.
Fig. 13 Single inclusion microstructure under pure shear loading: smoothed Von Mises stress fields $\left[\mathrm{N} / \mathrm{m}^{2}\right]$ in the sense of Eq.(11) around the optimized holes for target volumes of 20, 40,60 and $80 \mathrm{~m}^{3}$ with the objective of minimizing the local Von Mises stress.

hole volumes. They are compared against the HashinShtrikman bounds. Only the stress designs are considered here. In plane elasticity, the effective shear modulus $\mu^{H}$ can be recollected from the homogenized coefficients $H_{i j}^{H}$ and is given as:

$\mu^{H}=\frac{E^{H}}{2\left(1+\nu^{H}\right)}$.

For two-phase materials, in the case of plane elasticity and one material being void, the Hashin-Shtrikman bounds are given as a function of the microstructure density $\rho$, the bulk and the shear modulus of the solid phase $\kappa_{\text {solid }}$ and $\mu_{\text {solid }}[44]$, [5]:

$0 \leq \mu^{H} \leq \frac{\rho \kappa_{\text {solid }} \mu_{\text {solid }}}{(1-\rho)\left(\kappa_{\text {solid }}+2 \mu_{\text {solid }}\right)+\kappa_{\text {solid }}}$.

The comparison is summarized in Fig.(14). It shows that the optimized stress designs are suboptimal and so unable to saturate the Hashin-Shtrikman upper bound.

To further validate the choice of an initial combination of two superellipses, the performance of the optimized designs is evaluated in terms of maximal Von Mises stress achieved for different initial shapes. As in the previous example, a circle and an ellipse are considered. For growing hole volume, the maximum Von Mises stress for each initial shape is presented in Table 4. No results are provided for the circle and the ellipse for a target hole volume of $80 \mathrm{~m}^{3}$ as such a high volume can not be reached while remaining inside the RVE. As 


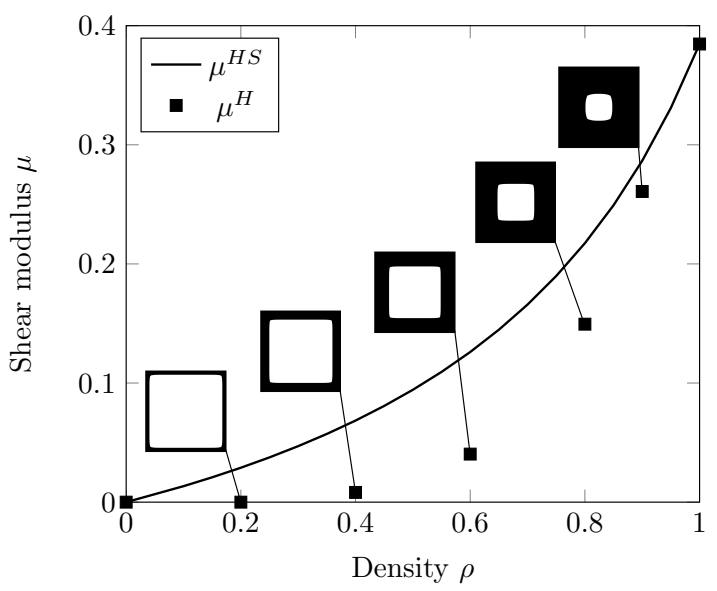

Fig. 14 Single inclusion microstructure under pure shear loading: comparison of the shear modulus obtained numerically $\mu^{H}$ with the Hashin-Shtrikman bounds $\mu^{H S}$.

expected, the maximum Von Mises stress is lower when the combination of two superellipses is used as the optimized shape is, in this case, a square with rounded sides.

Table 4 Single inclusion microstructure under pure shear loading: influence of the initial shape on the maximum Von Mises stress achieved.

\begin{tabular}{l|cccc}
$\sigma_{\max }^{\mathrm{VM}}\left[N / \mathrm{m}^{2}\right]$ for $\backslash V_{\max }\left[\mathrm{m}^{3}\right]$ & 20 & 40 & 60 & 80 \\
\hline Circle & 1.203 & 1.232 & 1.262 & $/$ \\
Ellipse & 1.203 & 1.232 & 1.262 & $/$ \\
2 Superellipses & 0.798 & 0.763 & 0.755 & 0.767
\end{tabular}

\subsection{Microstructures reinforced by stiff inclusions}

In this second example, microstructures reinforced by several stiff inclusions are investigated. Optimized shapes and locations of the inclusions are sought to minimize the local Von Mises stress in the microstructure. This problem is inspired from the work by Najafi et al. [29].

The inclusions are represented by circular shapes. Their radii $R_{i}$, as well as their locations $\left(X_{c i}, Y_{c i}\right)$ in the RVE, are used as design parameters. Overlapping of the inclusions is prohibited through the imposition of non penetrating constraints. Several initial configurations of the microstructure are considered. The inclusions are embedded in a soft matrix, i.e. $E_{\text {incl }} / E_{\text {matrix }}=100$. The RVE is loaded through a prescribed macroscopic strain field $\boldsymbol{\epsilon}=\left[\begin{array}{lll}2 & 1 & 0\end{array}\right]^{T}$. Both materials are characterized by an isotropic and linear elastic behavior. A plane stress state is assumed. The optimization problem to solve, for $n_{\text {incl }}$ inclusions, is formulated as follow:

$$
\begin{array}{lll}
\min _{\mathbf{s}} & \max _{e=1, \ldots, n_{e}}\left\{\sigma_{e}^{\mathrm{VM}}\right\} \\
\text { s.t. } & \sum_{j=1}^{n_{\text {incl }}} V_{j}-V_{\max }=0 & \\
& R_{k}+R_{l}+0.3-d_{k l} \leq 0, \quad k, l=1, \ldots, n_{\text {incl }} \\
& \underline{s}_{i} \leq s_{i} \leq \bar{s}_{i}, & i=1, \ldots, n
\end{array}
$$

where $d_{k l}$ is the distance between the $k^{t h}$ and the $l^{t h}$ inclusions.

This design problem is reformulated as detailed in Eq.(2). The stopping criteria are the relative variations of the design parameters and of the maximum Von Mises stress that have to be smaller than $10^{-3}$. All the parameters used through the optimization process are given in Table 5 .

Table 5 Multiple stiff inclusions microstructure: parameters.

\begin{tabular}{lll}
\hline Dimensions & {$[\mathrm{m}]$} & $c=10, t=1$ \\
Elastic moduli & {$\left[\mathrm{N} / \mathrm{m}^{2}\right]$} & $E_{\text {matrix }}=1, E_{\text {incl }}=100$ \\
Poisson's ratio & {$[-]$} & $\nu_{\text {matrix }}=\nu_{\text {incl }}=0.3$ \\
Hydrostatic loading & {$[-]$} & $\epsilon_{\text {shear }}=\left[\begin{array}{ll}2 \quad 1 & 0\end{array}\right]^{T}$ \\
Bound on $V_{\text {incl }}$ & {$\left[m^{2}\right]$} & $V_{\text {max }}=20$ \\
Bounds on design variables & {$[m]$} & $0.3 \leq R_{i} \leq 2$ \\
& {$[m]$} & $2 \leq X_{c i}, Y_{c i} \leq 8$ \\
Gauss points per subelement & $n_{g p}=7$ \\
Convergence criterion & $\Delta R_{i}, \Delta X_{c i}, \Delta Y_{c i} \leq 10^{-3}$ \\
& $\Delta \sigma_{\max }^{\mathrm{VM}} \leq 10^{-3}$ \\
Mesh & $100 \times 100$ bilinear Q4 \\
\hline
\end{tabular}

The inclusions are considered very stiff compared to the matrix, i.e. $E_{\text {incl }} / E_{\text {matrix }}=100$. Figure 15 shows the stress field in a microstructure made of five inclusions embedded in a soft matrix. The inclusions are highly stressed and their inner stress field is rather uniform. It results that the variations of the stress field within the inclusions are small and can be recollected with accuracy only resorting to very fine meshes. As the stress field within the inclusions is rather uniform and as failure is most likely to appear first in the soft matrix phase, we choose not to consider the stress field within the inclusions in the optimization process and the problem aims at minimizing the local Von Mises stress in the matrix. Therefore, the location of the inclusions is driven by the stress concentrations within the soft matrix.

The Von Mises stress fields for the initial and optimized designs around the inclusions are exposed in Fig.(16). One may remark that the maximum stress value is lower in the initial design; this is due to the fact that the initial configurations violate the constraint imposed on the inclusions volume.

Starting from several initial configurations, the resulting optimized designs can differ. For configurations 


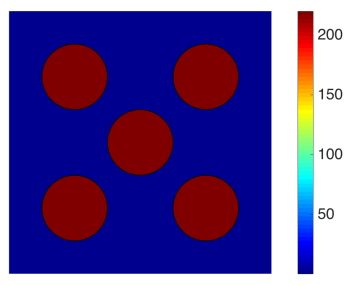

(a) Microstructure stress field.

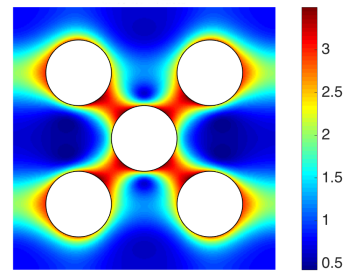

(b) Matrix stress field.

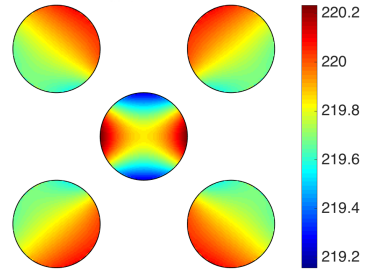

(c) Inclusions stress field.
Fig. 15 Multiple stiff inclusions microstructure: Smoothed Von Mises stress fields $\left[N / m^{2}\right]$ for $E_{\text {incl }} / E_{\text {matrix }}=100$ and $\epsilon=\left[\begin{array}{lll}2 & 1 & 0\end{array}\right]^{T}$ on a fine $300 \times 300 \mathrm{Q} 4$ mesh.

1 and 3, where no inclusion initially lies in the center of the RVE, final designs are rather similar. For configurations 2 and 3, where an inclusion initially lies in the center of the RVE, final designs are also very close. These designs are different and lead to different maximal Von Mises stress values. The non penetrating constraints induce a certain repulsion force between the inclusions. For designs 1 and 3, this repulsion prevents the displacement of the inclusions towards the center of the RVE. For designs 2 and 4, the inclusion lying in the center of the RVE limits the displacements of the other inclusions. They only move around the central one.

The convergence of the optimization process in terms of maximal Von Mises stress is given in Fig.(17) for the different initial configurations. The performance in terms of maximal Von Mises stress varies according to the initial configurations. One should remark that the objective function value oscillates. This happens as the objective is very sensitive to slight modifications of the interface, as small element subparts can be created. This sensitivity is related to the XFEM approximation and will be further investigated in future works.

After exhibiting these results, some important remarks have to be made:

- the symmetry of the initial configuration has a huge influence on the optimized designs. Starting from symmetric initial designs, it is more difficult for the optimization scheme to modify the position of the inclusions and so to break the symmetry.

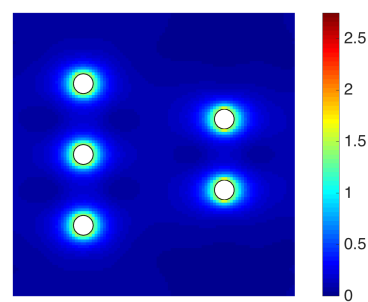

(a) Initial design 1 .

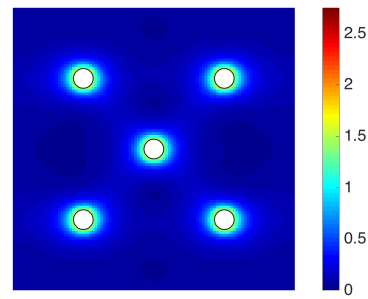

(c) Initial design 2 .

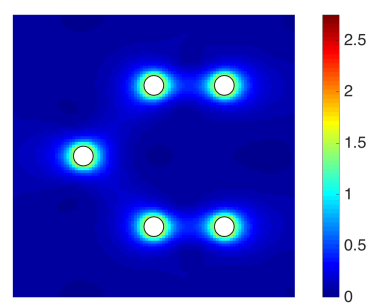

(e) Initial design 3 .

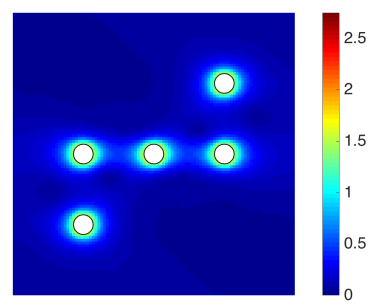

(g) Initial design 4.

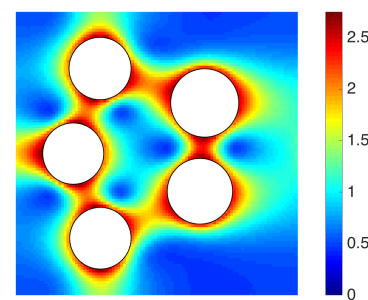

(b) Optimized design 1 .

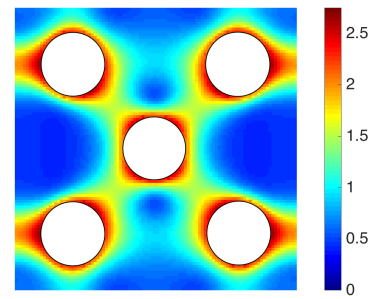

(d) Optimized design 2.

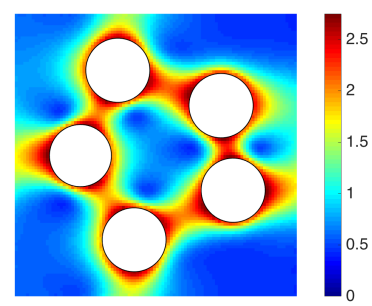

(f) Optimized design 3 .

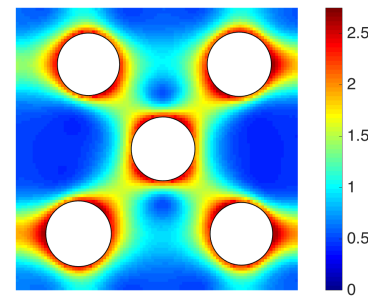

(h) Optimized design 4.
Fig. 16 Multiple stiff inclusions microstructure: smoothed Von Mises stress fields $\left[N / \mathrm{m}^{2}\right]$ in the sense of Eq.(11) for initial and optimized designs around the inclusions, for $E_{\text {incl }} / E_{\text {matrix }}=100$ and $\epsilon=\left[\begin{array}{lll}2 & 1 & 0\end{array}\right]^{T}$.

- the ratio between the stiffnesses of the inclusions and the matrix, $E_{\text {incl }} / E_{\text {matrix }}$, also plays a role. If this ratio is high, the inclusions are highly stressed and present a rather constant stress field. The movements of the inclusions through the optimization process are mainly driven by the stress field in the matrix. If the ratio is low, the magnitude of the stress field within the inclusions is rather close to the 


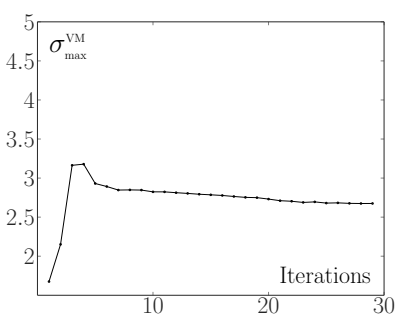

(a) Design 1.

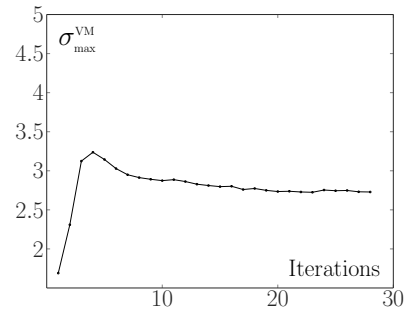

(c) Design 3

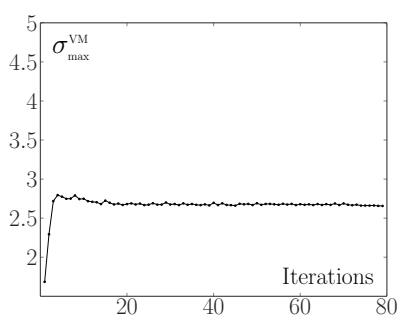

(b) Design 2 .

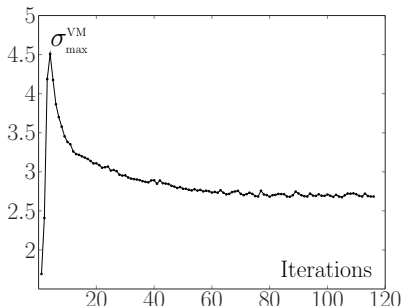

(d) Design 4.
Fig. 17 Multiple stiff inclusions microstructure: convergence in terms of maximal Von Mises stress for the different initial configurations for $E_{\text {incl }} / E_{\text {matrix }}=100$ and $\epsilon=\left[\begin{array}{lll}2 & 1 & 0\end{array}\right]^{T}$.

one existing in the matrix. This is shown in Fig.(18) where the same configuration as in Fig.(15) is considered but for $E_{\text {incl }} / E_{\text {matrix }}=2$. The stress field within the inclusions might then have a larger influence on their optimal locations.

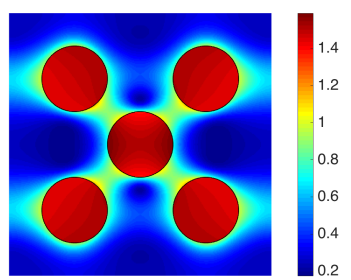

(a) Microstructure stress field.

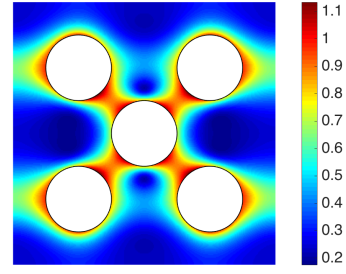

(b) Matrix stress field.

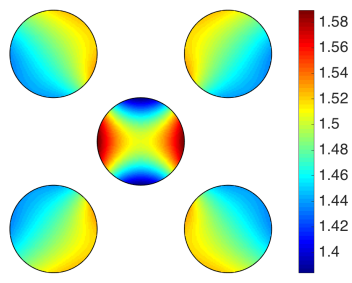

(c) Inclusions stress field.
Fig. 18 Multiple stiff inclusions microstructure: smoothed Von Mises stress fields $\left[N / \mathrm{m}^{2}\right]$ for $E_{\text {incl }} / E_{\text {matrix }}=2$ and $\epsilon=$ $\left[\begin{array}{lll}2 & 1 & 0\end{array}\right]^{T}$ on a fine $300 \times 300 \mathrm{Q} 4$ mesh.

- the symmetry of the loading also influences the optimized designs. A symmetric load case combined with an initial symmetric microstructure can prevent the modifications and movements of the inclusions in the RVE.

Finally, a more general remark is formulated on the evaluation of the local stress field resorting to homogenization procedures. Performing the optimization of microstructures while considering stress constraints, it is critical to know whether the chosen homogenization procedure provides an accurate indication of the strength of the composite in practice. This issue was recently addressed by Coelho et al. [9] who investigate whether homogenization local stress predictions are in agreement with numerical stress evaluations in composites build from the repetition of the unit cell in the three directions of space. Their study suggests that a low scale factor, i.e. a low number of repetition of the unit cell, is sufficient to replace the non-homogeneous composite by the equivalent homogeneous materials. While beyond the scope of this paper, this issue is of significant importance and should be further investigated in future works.

\section{Conclusions}

The present work has provided a flexible and robust approach to perform shape optimization of bimaterial microstructures minimizing their local stress field. The approach is based on a level set representation of the geometry and an XFEM discretization. While the level set representation allowed dealing with complex geometries and handling large shape modifications, the XFEM was chosen to avoid heavy remeshing operations inherent to classical shape optimization. Considering periodic materials built from the repetition of a RVE, the homogenization theory is applied to impose macroscopic strain fields and periodic boundary conditions to the considered RVE. Numerical homogenization techniques were adapted to fit the selected XFEM-level set framework. To perform shape optimization with stress objectives or constraints, the scope of the analytical approach to the sensitivity analysis, developed in previous work by the authors [31], was extended. The derivatives of the stress values were evaluated analytically starting from their discretized expressions and deriving them with respect to the design parameters. The different terms involved in the sensitivities were detailed.

Working with geometric features as design parameters, the developed approach is illustrated on some classical 2D shape optimization problems. The shape of holed microstructures is optimized to minimize the Von Mises stress field induced by hydrostatic or pure shear loadings. Results obtained are very similar to the 
Vigdergauz microstructure. The developed approach is able to tackle multiple inclusions problems to find the optimal design of a bimaterial microstructure minimizing its Von Mises stress field.

Ongoing work aims on the one hand, at considering other strategies to control the local stress field. In particular, the minimization of stress ratios can be considered resorting to different failure stresses so as to simultaneously deal with the stress in the matrix and in the inclusions. On the other hand, the optimization problem can be modified to combine the local stress field control with some restriction on the stiffness. The design problem would then focus on maximizing the stiffness while minimizing the local stress field within the microstructure. To allow a greater freedom in the design, nodal level set values could be used as design parameters. Finally, the work should be further extended to tackle some non linear behaviors of materials within the microstructure.

Acknowledgements The authors would like to thank E. Andreassen for the help with the homogenization implementation and for providing the code related to [3].

\section{References}

1. Allaire, G., Jouve, F., Toader, A.M.: A level-set method for shape optimization. Comptes Rendus Mathematique 334(12), 1125-1130 (2002)

2. Allaire, G., Kohn, R.V.: Optimal design of minimum compliance. European Journal of Mechanics A 12, 839878 (1993)

3. Andreassen, E., Schousboe Andreasen, C.: How to determine composite material properties using numerical homogenization. Computational Materials Science 83, 488-495 (2014)

4. Bendsøe, M., Kikuchi, N.: Generating optimal topologies in structural design using a homogenization method. Computer Methods in Applied Mechanics Engineering 71, 197-224 (1988)

5. Bendsøe, M., Sigmund, O.: Material interpolation schemes in topology optimization. Archive of Applied Mechanics 69, 635-654 (1999)

6. Bendsøe, M.P.: Optimal shape design as a material distribution problem. Structural Optimization 1, 193-202 (1989)

7. Bensoussan, A., Lions, J.L., Papanicolaou, G.: Asymptotic analysis for periodic structures. North-Holland (1978)

8. Bordas, S., Duflot, M.: Derivative recovery and a posteriori error estimate for extended finite elements. Computer Methods in Applied Mechanics and Engineering 196, 3381-3399 (2007)

9. Coelho, P.G., Reis, R.A., Guedes, J.M.: Convergence analysis of stress fields to homogenization predictions in optimal periodic composite design. In: Proceedings of the VII European Congress on Computational Methods in Applied Sciences and Engineering. Crete Island, Greece (2016)
10. Diaz, A.R., Kikuchi, N.: Solutions to shape and topology eigenvalue optimization problems using a homogenization method. International Journal for Numerical Methods in Engineering 35, 1487-1502 (1992)

11. van Dijk, N.P., Maute, K., Langelaar, M., van Keulen, F.: Level-set methods for structural topology optimization: a review. Structural and Multidisciplinary Optimization 48, 437-472 (2013)

12. Duysinx, P., Bends $\varnothing$ e, M.: Topology optimization of continuum structures with local stress constraints. International Journal for Numerical Methods in Engineering 43, 1453-1478 (1998)

13. Gibiansky, L., Sigmund, O.: Multiphase composites with extremal bulk modulus. Journal of the Mechanics and Physics of Solids 48, 461-498 (2000)

14. Grabovsky, Y., Kohn, R.V.: Microstructures minimizing the energy of a two phase elastic composite in two space dimensions. ii: The vigdergauz microstructure. Journal of the Mechanics and Physics of Solids 43(6), 949-972 (1995)

15. Guedes, J.M., Kikuchi, N.: Preprocessing and postprocessing for materials based on the homogenization method with adaptive finite element methods. Computer Methods in Applied Mechanics and Engineering 83, 143198 (1990)

16. Guest, J., Prévost, J.H.: Optimizing multifunctional materials: Design of microstructures for maximized stiffness and fluid permeability. International Journal of Solids and Structures 43, 7028-7047 (2006)

17. Hashin, Z., Shtrikman, S.: A variational approach to the theory of the elastic behaviour of multiphase materials. Journal of the Mechanics and Physics of Solids 11, 127140 (1963)

18. Hassani, B., Hinton, E.: A review of homogenization and topology optimization i-homogenization theory for media with periodic structure. Computers and Structures 69, 707-717 (1997)

19. Hassani, B., Hinton, E.: A review of homogenization and topology optimization ii-analytical and numerical solution of homogenization equations. Computers and Structures 69, 719-738 (1998)

20. Hassani, B., Hinton, E.: A review of homogenization and topology optimization iii-topology optimization using optimality criteria. Computers and Structures 69, 739-756 (1998)

21. Krizek, M., Neittaanmaki, P.: On superconvergence techniques. Acta Applicandae Mathematicae 9, 175-198 (1987)

22. Lipton, R.: Design of functionally graded composite structures in the presence of stress constraints. International Journal of Solids and Structures 39, 2575-2586 (2002)

23. Lipton, R., Stuebner, M.: Optimization of composite structures subject to local stress constraints. Computer Methods in Applied Mechanics and Engineering 196, 6675 (2006)

24. Lipton, R., Stuebner, M.: Optimal design of composite structures for strength and stiffness: an inverse homogenization approach. Structural and Multidisciplinary Optimization 33, 351-362 (2007)

25. Mlejnek, H.P., Schirrmacher, R.: An engineer's approach to optimal material distribution and shape finding. Computer Methods in Applied Mechanics and Engineering 106(1), 1-26 (1993)

26. Moës, N., Cloirec, M., Cartaud, P., J.-F., R.: A computational approach to handle complex microstructure geometries. Computer Methods in Applied Mechanics and Engineering 192, 3163-3177 (2003) 
27. Moës, N., Dolbow, J., Belytschko, T.: A finite element method for crack growth without remeshing. International Journal for Numerical Methods in Engineering 46, 131-150 (1999)

28. Moës, N., Gravouil, A., Belytschko, T.: Non-planar 3d crack growth by the extended finite element and level sets-part i: Mechanical model. International Journal for Numerical Methods in Engineering 53, 2549-2568 (2002)

29. Najafi, A.R., Safdari, M., Tortorelli, D.A., Geubelle, P.H.: A gradient-based shape optimization scheme using an interface-enriched generalized fem. Computer Methods in Applied Mechanics and Engineering 296, 1-17 (2015)

30. Najafi, A.R., Safdari, M., Tortorelli, D.A., Geubelle, P.H.: Material design using a nurbs-based shape optimization scheme. In: Proceedings of the 57th AIAA/ASCE/AHS/ASC Structures, Structural Dynamics, and Materials Conference. San Diego, California (2016)

31. Noël, L., Van Miegroet, L., Duysinx, P.: Analytical sensitivity analysis using the extended finite element method in shape optimization of bimaterial structures. International Journal for Numerical Methods in Engineering 107(8), 669-695 (2016)

32. Osher, S., Sethian, J.A.: Fronts propagating with curvature-dependent speed: Algorithms based on hamilton-jacobi formulations. Journal of Computational Physics 79(1), 12-49 (1988)

33. Procházka, P.P., Válek, M.: Shape optimization of fibers in frc. Acta Geodynamica et Geomaterialia 10(1(169)), $111-120(2013)$

34. Ródenas, J.J., González-Estrada, O.A., Tarancón, J.E., Fuenmayor, F.J.: A recovery-type error estimator for the extended finite element method based on singular+smooth stress field splitting. International Journal for Numerical Methods in Engineering 76, 545-571 (2008)

35. Sanchez-Palencia, E.: Homogenization method for the study of composite media. In: F. Verhulst (ed.) Asymptotic Analysis II, Lecture Notes in Mathematics, vol. 985, pp. 192-214. Springer Berlin Heidelberg (1983)

36. Schousboe Andreasen, C., Andreassen, E., Søndergaard Jensen, J., Sigmund, O.: On the realization of the bulk modulus bounds for two-phase viscoelastic composites. Journal of the Mechanics and Physics of Solids 63, 228-241 (2014)

37. Sigmund, O.: Materials with prescribed constitutive parameters: An inverse homogenization problem. International Journal of Solids and Structures 31(17), 2313-2329 (1994)

38. Sigmund, O.: Tailoring materials with prescribed elastic properties. Mechanics of Materials 20, 351-368 (1995)

39. Sigmund, O., Torquato, S.: Design materials with extreme thermal expansion using a three-phase topology optimization method. Journal of the Mechanics and Physics of Solids 45(6), 1037-1067 (1997)

40. Suquet, P.: Une méthode duale en homogénéisation : application aux milieux élastiques. Journal de Mécanique Théorique et Appliquée pp. 79-98 (1982)

41. Suzuki, K., Kikuchi, N.: Homogenization method for shape and topology optimization. Computer Methods in Applied Mechanics and Engineering 93, 291-318 (1991)

42. Svanberg, K.: The method of moving asymptotes-a new method for structural optimization. International Journal for Numerical Methods in Engineering 24, 359-373 (1987)

43. Torquato, S.: Random Heterogeneous Materials/Microstructure and Macroscopic Properties. Springer, New York (2002)
44. Torquato, S., Gibiansky, L.V., Silva, M.J., Gibson, L.: Effective mechanical and transport properties of cellular solids. International Journal of Mechanical Sciences 40, 71-82 (1998)

45. Van Miegroet, L.: Generalized shape optimization using xfem and level set description. Ph.D. thesis, University of Liège, Liège (2012)

46. Van Miegroet, L., Duysinx, P.: Stress concentration minimization of $2 \mathrm{~d}$ filets using $\mathrm{x}$-fem and level set description. Structural and Multidisciplinary Optimization 33(4-5), 425-438 (2007)

47. Vigdergauz, S.: Two-dimensional grained composites of extreme rigidity. ASME Journal of Applied Mechanics 61, 390-394 (1994)

48. Vigdergauz, S.: The effective properties of a perforated elastic plate numerical optimization by genetic algorithm. International Journal of Solids and Structures 38, 85938616 (2001)

49. Vigdergauz, S.: Genetic algorithm perspective to identify energy optimizing inclusions in an elastic plate. International Journal of Solids and Structures 38, 6851-6867 (2001)

50. Wang, M.Y., Wang, X., Guo, D.: A level set method for structural topology optimization. Computer Methods in Applied Mechanics and Engineering 192(1-2), 227-246 (2003)

51. Weihong, Z., Fengwen, W., Gaoming, D., Shiping, S.: Topology optimal design of material microstructures using strain energy-based method. Chinese Journal of Aeronautics 20, 320-326 (2007)

52. Zhou, Y., Li, C., Mason, J.J.: Shape optimization of randomly oriented short fibers for bone cement reinforcements. Materials Science and Engineering: A 393(1-2), 374-381 (2005)

53. Zienkiewicz, O.C., Zhu, J.Z.: The superconvergent patch recovery a posteriori error estimates. part 1: The recovery technique. International Journal for Numerical Methods in Engineering 33, 1331-1364 (1992) 